\title{
ON THE NEGATIVE PELL EQUATION $y^{2}=45 x^{2}-11$
}

\author{
M.A.Gopalan ${ }^{1}$, S.Vidhyalakshmi ${ }^{2}$, V.Pandichelvi ${ }^{3}$, \\ P.Sivakamasundari ${ }^{4}$, C.Priyadharsini ${ }^{5}$ \\ ${ }^{1,2}$ Professor, Dept. of Mathematics, SIGC, Trichy, \\ email: mayilgopalan@gmail.com, vidhyasigc@gmail.com \\ ${ }^{3}$ Asst.Professor, Dept. of Mathematics, UDC, Trichy,email:bbasmahesh@gmail.com \\ ${ }^{4}$ Guest Lecturer, Dept.of Mathematics, BDUCC, Lalgudi,Trichy, email:kaneeska.s@gmail.com \\ ${ }^{5}$ M.Phil Scholar, Dept. of Mathematics, SIGC, Trichy,email:priyamat16@gmail.com
}

KEYWORDS: Binary quadratic, hyperbola, parabola, integral solutions, pell equation.

\begin{abstract}
The binary quadratic equation represented by the negative pellian $y^{2}=45 x^{2}-11$ is analyzed for its distinct integer solutions.A few interesting relations among the solutions are also given. Further, employing the solutions of the above hyperbola, we have obtained solutions of other choices of hyperbolas, parabolas and special Pythagorean triangle.
\end{abstract}

\section{INTRODUCTION:}

Diophantine equation of the form $y^{2}=D x^{2}+1$, where $\mathrm{D}$ is a given positive square-free integer is known as pell equation and is one of the oldest Diophantine equation that has interesting mathematicians all over the world, since antiquity, J.L.Lagrange proved that the positive Pell equation $\mathrm{y}^{2}=D x^{2}+1$ has infinitely many distinct integer solutions whereas the negative pell equation $\mathrm{y}^{2}=D x^{2}-1$ does not always have a solution. In [1], an elementary proof of a ceriterium for the solvability of the pell equation $\mathrm{x}^{2}-D y^{2}=-1$ where $\mathrm{D}$ is any positive non-square integer has been presented by R.A.Mollin and Anitha Srinivasan. For examples the equations $\mathrm{y}^{2}=3 x^{2}-1, y^{2}=7 x^{2}-4$ have no integer solutions whereas $\mathrm{y}^{2}=65 x^{2}-1, y^{2}=202 x^{2}-1$ have integer solutions. In this context, one may refer \{[2] E.E.Whitford, [3] S. Ahmet Tekcan et al., [4] Ahmet Tekcan, [5] Merve Guney, [6] V.Sangeetha et al., [7,8,11,12,13] M.A.Gopalan, [9,10] K.Meena et al.,\}. More specifically, one may refer "The On-line Encyclopedia of integer sequences" (A031396, A130226, A031398) for values of D for which the negative pell equation $\mathrm{y}^{2}=D x^{2}-1$ is solvable or not. In this communication, the negative Pell equation given by $\mathrm{y}^{2}=45 x^{2}-11$ is considered and infinitely many integer solutions are obtained. A few interesting relations among the solutions are presented.

\section{METHOD OF ANALYSIS:}

The negative pell equation representing hyperbola under consideration is

$$
y^{2}=45 x^{2}-11
$$

whose smallest positive integer solution is $x_{0}=2, y_{0}=13$

To obtain the other solutions of (1),consider the pell equation $y^{2}=45 x^{2}+1$

whose general solution $\left(\tilde{x}_{n}, \tilde{y}_{n}\right)[14]$ is given by $\tilde{x}_{n}=\frac{1}{2 \sqrt{45}} g_{n}, \tilde{y}_{n}=\frac{1}{2} f_{n}$ 
where, $\left\{f_{n}=(161+24 \sqrt{45})^{n+1}+(161-24 \sqrt{45})^{n+1} ; g_{n}=(161+24 \sqrt{45})^{n+1}-(161-24 \sqrt{45})^{n+1}\right\}$

Applying Brahamagupta Lemma[16] between $\left(x_{0}, y_{0}\right)$ and $\left(\tilde{x}_{n}, \tilde{y}_{n}\right)$, the other integer solutions of (1) are given by $90 x_{n+1}=90 f_{n}+13 \sqrt{45} g_{n}$

$$
2 y_{n+1}=13 f_{n}+2 \sqrt{45} g_{n}
$$

Some numerical examples of $x \& y$ satisfying (1) are given in the table below

\begin{tabular}{|c|c|c|}
\hline$n$ & $x_{n}$ & $y_{n}$ \\
\hline 0 & 2 & 13 \\
\hline 1 & 634 & 4253 \\
\hline 2 & 204146 & 1369453 \\
\hline 3 & 65734378 & 440959613 \\
\hline 4 & 21166265570 & 141987625933 \\
\hline
\end{tabular}

\section{Observations:}

From the above table, we observe some interesting relations among the solutions which are presented below

1) The $x$-values are even whereas the $y$-values are odd

2) The recurrence relations satisfied by the solutions of (1) are given by

$$
\begin{aligned}
& x_{n+3}-322 x_{n+2}+x_{n+1}=0 \\
& y_{n+3}-322 y_{n+2}+y_{n+1}=0
\end{aligned}
$$

3) $x_{n+3}=322 x_{n+2}-x_{n+1}$

4) $24 y_{n+1}=x_{n+2}-161 x_{n+1}$

5) $24 y_{n+2}=161 x_{n+2}-x_{n+1}$

6) $24 y_{n+3}=51841 x_{n+2}-161 x_{n+1}$

7) $51841 x_{n+1}=x_{n+3}-7728 y_{n+1}$

8) $25920 x_{n+2}=3864 y_{n+2}-24 y_{n+1}$

9) $25920 x_{n+2}=24 y_{n+3}-3864 y_{n+2}$

10) $x_{n+2}=161 x_{n+3}-24 y_{n+3}$

11) $51840 x_{n+2}=24 y_{n+3}-24 y_{n+1}$ 
12) $161 x_{n+2}=x_{n+3}-24 y_{n+2}$

13) $51841 x_{n+2}=161 x_{n+3}-24 y_{n+1}$

14) $25920 x_{n+1}=24 y_{n+2}-3864 y_{n+1}$

15) $51841 x_{n+2}^{2}=161 x_{n+3} x_{n+2}-24 y_{n+1} x_{n+2}$

16) $51841 x_{n+1}^{2}=x_{n+1} x_{n+3}-7728 x_{n+1} y_{n+1}$

17) $24 y_{n+1} x_{n+1}=3864 y_{n+2} x_{n+1}-25920 x_{n+1} x_{n+2}$

18) $3864 y_{n+1} x_{n+2}=24 y_{n+2} x_{n+2}-25920 x_{n+1} x_{n+2}$

19) $\frac{180 x_{2 n+2}-26 y_{2 n+2}+22}{11}$ is a perfect square

Proof: Eliminating $g_{n}$ between (4) and (5), we get

$180 x_{n+1}-26 y_{n+1}=11 f_{n}$

Similarly, Eliminating $f_{n}$ between (4) and (5), we get

$4 \sqrt{45} y_{n+1}-26 \sqrt{45} x_{n+1}=11 g_{n}$

Replacing $n$ by $2 n+1$ in (8), It is seen that

$180 x_{2 n+2}-26 y_{2 n+2}=11\left[f_{n}^{2}-2\right]$

Therefore, $\frac{180 x_{2 n+2}-26 y_{2 n+2}+22}{11}$ is a perfect square

Similarly, each of the following expressions is a perfect square

i) $\frac{1369453 x_{2 n+2}-13 x_{2 n+4}+85008}{42504}$

ii) $\frac{1369453 x_{2 n+3}-4253 x_{2 n+4}+264}{132}$

iii) $\frac{57060 x_{2 n+2}-26 y_{2 n+3}+3542}{1771}$

iv) $\frac{18373140 x_{2 n+2}-26 y_{2 n+4}+1140502}{570251}$

v) $\frac{4253 x_{2 n+2}-13 x_{2 n+3}+264}{132}$

vi) $\frac{180 x_{2 n+3}-8506 y_{2 n+2}+3542}{1771}$ 
viii) $\frac{57060 x_{2 n+3}-8506 y_{2 n+3}+22}{11}$

20) $\frac{180 x_{3 n+3}-26 y_{3 n+3}+10626}{11}$ is a cubical integer

Proof:

Replacing $n$ by $3 n+2$ in (8), it is seen that

$$
\frac{180 x_{3 n+3}-26 y_{3 n+3}+10626}{11} \text { is a cubical integer }
$$

\section{REMARKABLE OBSERVATIONS:}

I: $\quad$ It is seen that $f_{n}^{2}-g_{n}^{2}=4$

Define $\left(X=180 x_{n+1}-26 y_{n+1}, Y=4 \sqrt{45} y_{n+1}-26 \sqrt{45} x_{n+1}\right)$

Therefore, $f_{n}=\frac{X}{11}, g_{n}=\frac{Y}{11}$

Substituting the above values of $\left(f_{n}, g_{n}\right)$ in (10), we have

$X^{2}-Y^{2}=484$ which represents a hyperbola.

Similarly, employing linear combinations among the solutions of (1), one may generate integer solutions for other choices of hyperbolas which are presented in the table 1 below

TABLE: 1

\begin{tabular}{|c|c|c|}
\hline S.NO & HYPERBOLA & $(X, Y)$ \\
\hline 1 & $X^{2}-Y^{2}=7226360064$ & $\left(1369453 x_{n+1}-13 x_{n+3}, \frac{90 x_{n+3}-9186570 x_{n+1}}{\sqrt{45}}\right)$ \\
\hline 2 & $X^{2}-Y^{2}=484$ & $\left(180 x_{n+1}-26 y_{n+1}, \frac{180 y_{n+1}-1170 x_{n+1}}{\sqrt{45}}\right)$ \\
\hline 3 & $X^{2}-Y^{2}=69696$ & $\left(1369453 x_{n+2}-4253 x_{n+3}, \frac{28530 x_{n+3}-9186570 x_{n+2}}{\sqrt{45}}\right)$ \\
\hline 4 & $X^{2}-Y^{2}=1300744812004$ & $\left(18373140 x_{n+1}-26 y_{n+3}, \frac{180 y_{n+3}-123250770 x_{n+1}}{\sqrt{45}}\right)$ \\
\hline 5 & $X^{2}-Y^{2}=12545764$ & $\left(57060 x_{n+1}-26 y_{n+2}, \frac{180 y_{n+3}-382770 x_{n+1}}{\sqrt{45}}\right)$ \\
\hline 6 & $X^{2}-Y^{2}=69696$ & $\left(4253 x_{n+1}-13 x_{n+2}, \frac{90 x_{n+2}-28530 x_{n+1}}{\sqrt{45}}\right)$ \\
\hline
\end{tabular}




\begin{tabular}{|c|c|c|}
\hline 7 & $X^{2}-Y^{2}=12545764$ & $\left(180 x_{n+2}-8506 y_{n+1}, \frac{57060 y_{n+1}-1170 x_{n+2}}{\sqrt{45}}\right)$ \\
\hline 8 & $X^{2}-Y^{2}=484$ & $\left(57060 x_{n+2}-8506 y_{n+2}, \frac{57060 y_{n+2}-382770 x_{n+2}}{\sqrt{45}}\right)$ \\
\hline
\end{tabular}

II. Define $\left(X=180 x_{2 n+2}-26 y_{2 n+2}+22, Y=4 \sqrt{45} y_{n+1}-26 \sqrt{45} x_{n+1}\right)$

Therefore, $f_{n}^{2}=\frac{X}{11}, g_{n}^{2}=\frac{Y^{2}}{11^{2}}$

Substituting the above values of $\left(f_{n}^{2}, g_{n}^{2}\right)$ in (10), we have

$Y^{2}=11 X-484$ which represents a parabola.

Similarly, Employing linear combinations among the solutions of (1), one may generate integer solutions for other choices of parabolas which are presented in the table 2 below

TABLE: 2

\begin{tabular}{|c|c|c|}
\hline S.NO & PARABOLA & $(X, Y)$ \\
\hline 1 & $Y^{2}=42504 X-7226360064$ & $\left(1369453 x_{2 n+2}-13 x_{2 n+4}+85008, \frac{90 x_{n+3}-9186570 x_{n+1}}{\sqrt{45}}\right)$ \\
\hline 2 & $Y^{2}=11 X-484$ & $\left(180 x_{2 n+2}-26 y_{2 n+2}+22, \frac{180 y_{n+1}-1170 x_{n+1}}{\sqrt{45}}\right)$ \\
\hline 3 & $Y^{2}=132 X-69696$ & $\left(1369453 x_{2 n+3}-4253 x_{2 n+4}+264, \frac{28503 x_{n+3}-9186570 x_{n+2}}{\sqrt{45}}\right)$ \\
\hline 4 & $Y^{2}=570251 X-1300744812004$ & $\left(\begin{array}{l}18373140 x_{2 n+2}-26 y_{2 n+4}+1140502 \\
\frac{180 y_{n+3}-123250770 x_{n+1}}{\sqrt{45}}\end{array}\right)$ \\
\hline 5 & $Y^{2}=1771 X-12545764$ & $\left(57060 x_{2 n+2}-26 y_{2 n+3}+3542, \frac{180 y_{n+3}-382770 x_{n+1}}{\sqrt{45}}\right)$ \\
\hline 6 & $Y^{2}=132 X-69696$ & $\left(4253 x_{2 n+2}-13 x_{2 n+3}+264, \frac{90 x_{n+2}-28530 x_{n+1}}{\sqrt{45}}\right)$ \\
\hline 7 & $Y^{2}=1771 X-1254564$ & $\left(180 x_{2 n+3}-8506 y_{2 n+2}+3542, \frac{57060 y_{n+1}-1170 x_{n+2}}{\sqrt{45}}\right)$ \\
\hline 8 & $Y^{2}=11 X-484$ & $\left(57060 x_{2 n+3}-8506 y_{2 n+3}+22, \frac{57060 y_{n+2}-382770 x_{n+2}}{\sqrt{45}}\right)$ \\
\hline
\end{tabular}


III. Let $p, q ; p>q>0$ be the generators of the Pythagorean triangle $T(\alpha, \beta, \gamma)$, where $\alpha=2 p q, \beta=p^{2}-q^{2}, \gamma=p^{2}+q^{2}, p>q>0$. Let A,P represent the area and perimeter of $\mathrm{T}$ respectively, where $A=p q\left(p^{2}-q^{2}\right), P=2 p(p+q)$

Note that $\quad \gamma-\beta=2 q^{2} ; \gamma-\alpha=(p-q)^{2}$

Therefore, $2(\gamma-\alpha)=45(\gamma-\beta)-22$

gives $(p-q)^{2}=45 q^{2}-11$

Comparing (12) with (1), we have $p=x_{n+1}+y_{n+1}, q=x_{n+1}$

Thus, the Pythagorean triangle T with generators $p, q$ given by (13) is such that

$$
2 \alpha-45 \beta+43 \gamma=22
$$

In a similar manner, the other relations for the Pythagorean triangle $\mathrm{T}$ are presented below.
a) $47 \beta-45 \gamma-\frac{8 A}{P}=-22$
b) $2 \alpha-\frac{4 A}{P}+\beta=\left(2 x_{n+1}+y_{n+1}\right)^{2}$
c) $\quad \gamma-\frac{4 A}{P}-\alpha+\beta=2 y_{n+1}^{2}$
d) $\frac{2 A}{P}=x_{n+1} y_{n+1}$

Each of the following expressions is a nasty number [15]
1. $6\left(\beta-\frac{4 A}{P}\right)$
2. $6\left(2 \alpha-\frac{4 A}{P}+\beta\right)$
3. $3\left(\gamma-\frac{4 A}{P}-\alpha+\beta\right)$

\section{CONCLUSION:}

In this paper, we have presented infinitely many integer solutions for the hyperbola represented by the negative Pell equation $y^{2}=45 x^{2}-11$. As the binary quadratic Diophantine equations are rich in variety, one may search for the other choices of negative Pell equations and determine their integer solutions along with suitable properties. 


\section{REFERENCES:}

[1]. R.A.Mollin and Anitha Srinivasan "A Note On The Negative Pell Equation", International Journal of Algebra, Vol4, no. 19, 2010, 919-922.

[2]. E.E.Whitford, "Some Solutions of The Pellian Equations $x^{2}-A y^{2}= \pm 4$ " JSTOR: Annals of Mathematics, Second Series,. Vol. no. 1, (1913-1914) (157-160).

[3]. S. Ahmet Tekcan, Betw Gezer And Osman Bizim, "On The Integer Solutions of The Pell Equation $x^{2}-d y^{2}=2^{t}$, , World Academy of Science, Engineering and Technology 1, 2007 (522526).

[4]. Ahmet Tekcan " The Pell Equation $x^{2}-\left(k^{2}-k\right) y^{2}=2^{t}$ ", World Academy of Science, Engineering and Technology, 19, $2008,(697-701)$.

[5]. Merve Guney, "Solutions of the pell equations $x^{2}-\left(a^{2} b^{2}+2 b\right) y^{2}=2^{t}$, when $N \in( \pm 1, \pm 4)$ ", Mathematica Aterna, Vol 2, no.7,2012, (629-638).

[6]. V.Sangeetha, M.A.Gopalan and Manju Somanath, "On the Integral Solutions of the pell Equation $x^{2}=13 y^{2}-3^{t}$ ", International Journal of Applied Mathematical Research, ,Vol 3 issue 1, $2014(58-61)$.

[7]. M.A.Gopalan, G.Sumathi, S.vidhyalakshmi , "Observations on the hyperbola $x^{2}=19 y^{2}-3^{t}$ ", Scholars Journal of the Engineering and Technology. Vol:2(2A), 2014,152-155.

[8]. M.A.Gopalan, S.Vidhyalakshmi and A.Kavitha, "On The Integral Solution of the Binary Quadratic Equation $x^{2}=15 y^{2}-11^{t}$, Scholars Journal of the Engineering and Technology, Vol 2(2A), 2014,156-158.

[9]. K.Meena, M.A.Gopalan, R.Karthika, On the negative pell equation $y^{2}=10 x^{2}-6$, IJMRD, VOL 2(12), 2015, 390-392

[10]. K.Meena, M.A.Gopalan, E.Bhuvaneshwari, On the negative pell equation $y^{2}=60 x^{2}-15$, Scholars Bulletin, VOL 1(11), 2015, 310-316

[11]. M. A. Gopalan, S. Vidhyalakshmi, J. Shanthi, D. Kanaka, On the negative Pell Equation $y^{2}=15 x^{2}-6$, SJPMS, Vol-2, Issue-2A,2015, PP:123-128.

[12]. M.A.Gopalan, R.Presenna, N.Thiruniraiselvi, "On the negative pell equation $y^{2}=24 x^{2}-87$ “, Proceedings of National Conference (UGC Sponseored) on recent developments on emerging fields in pure and applied mathematics, ReDeEM, March 2015, 138-144.

[13]. M.A.Gopalan, S.Vidhyalakshmi , T.R.Usha Rani, V. Kumari, “ Observations on the negative pellian $y^{2}=86 x^{2}-5$, International Journal of Applied Research; vol 1(3): 2015, 86-87.

[14]. L.J.Mordell., Diophantine equations, Academic Press, New York, 1969.

[15]. Bhatia, B.L. and Supriya Mohanty, Nasty numbers and their characterizations, Mathematical Education, 34-37, July-Sep., 1985.

[16]. Bhanumurthy, T.S., A modern introduction to Ancient Indian Mathematics, New Age International Publishers limited, New Delhi, 1995. 